\title{
Direct versus indirect bypass procedure for the treatment of ischemic moyamoya disease: results of an individualized selection strategy
}

\author{
Troels H. Nielsen, MD, PhD, ${ }^{1}$ Kumar Abhinav, FRCS(SN), ${ }^{1}$ Eric S. Sussman, MD, ${ }^{1}$ \\ Summer S. Han, PhD, ${ }^{1,2}$ Yingjie Weng, MHS, ${ }^{2}$ Teresa Bell-Stephens, RN, BSN, CNRN, ${ }^{1}$ \\ Jeremy J. Heit, MD, PhD, ${ }^{3}$ and Gary K. Steinberg, MD, PhD ${ }^{1}$ \\ ${ }^{1}$ Department of Neurosurgery and Stanford Stroke Center, Stanford University School of Medicine; ${ }^{2}$ Quantitative Sciences Unit, \\ Department of Medicine, Stanford University School of Medicine; and ${ }^{3}$ Department of Radiology, Neurolnterventional Radiology \\ Section, and Stanford Stroke Center, Stanford University School of Medicine, Stanford, California
}

\begin{abstract}
OBJECTIVE The only effective treatment for ischemic moyamoya disease (iMMD) is cerebral revascularization by an extracranial to intracranial bypass. The preferred revascularization method remains controversial: direct versus indirect bypass. The purpose of this study was to test the hypothesis that method choice should be personalized based on angiographic, hemodynamic, and clinical characteristics to balance the risk of perioperative major stroke against treatment efficacy.

METHODS Patients with iMMD were identified retrospectively from a prospectively maintained database. Those with mild to moderate internal carotid artery or $M_{1}$ segment stenosis, preserved cerebrovascular reserve, intraoperative $M_{4}$ segment anterograde flow $\geq 8 \mathrm{ml} / \mathrm{min}$, or the absence of frequent and severe transient ischemic attacks (TIAs) or stroke had been assigned to indirect bypass. The criteria for direct bypass were severe ICA or $\mathrm{M}_{1}$ segment stenosis or occlusion, impaired cerebrovascular reserve or steal phenomenon, intraoperative $\mathrm{M}_{4}$ segment retrograde flow or anterograde flow $<8 \mathrm{~m} / \mathrm{min}$, and the presence of frequent and severe TIAs or clinical strokes. The primary study endpoint was MRI-confirmed symptomatic stroke $\leq 7$ days postoperatively resulting in a decline in the modified Rankin Scale (mRS) score from preoperatively to 6 months postoperatively. As a secondary endpoint, the authors assessed 6 -month postoperative DSA-demonstrated revascularization, which was classified as $<1 / 3,1 / 3-2 / 3$, or $>2 / 3$ of the middle cerebral artery territory.
\end{abstract}

RESULTS One hundred thirty-eight patients with iMMD affecting 195 hemispheres revascularized in the period from March 2016 to June 2018 were included in this analysis. One hundred thirty-three hemispheres were revascularized with direct bypass and 62 with indirect bypass. The perioperative stroke rate was $4.7 \%$ and $6.8 \%$ in the direct and indirect groups, respectively $(p=0.36)$. Degree of revascularization was higher in the direct bypass group $(p=0.03)$. The proportion of patients improving to an mRS score $0-1$ (from preoperatively to 6 months postoperatively) tended to be higher in the direct bypass group, although the difference between the two bypass groups was not statistically significant $(p=0.27)$.

CONCLUSIONS The selective use of an indirect bypass procedure for IMMD did not decrease the perioperative stroke rate. Direct bypass provided a significantly higher degree of revascularization. The authors conclude that direct bypass is the treatment of choice for iMMD.

https://thejns.org/doi/abs/10.3171/2020.3.JNS192847

KEYWORDS cerebral revascularization; direct bypass; indirect bypass; ischemic stroke; moyamoya disease; vascular disorders

$\mathrm{M}$ OYAMOYA disease (MMD) is a progressive stenoocclusive disease of the distal portions of the internal carotid arteries (ICAs). The resulting progressive hypoperfusion promotes the formation of numerous collateral vessels on the base of the brain that give rise to the characteristic angiographic appearance of a "puff of smoke."' The disease can present with either ischemic manifestations (transient ischemic attacks [TIAs] or ischemic stroke) or hemorrhagic symptoms.

The only effective treatment to prevent future ischemic

ABBREVIATIONS DWI = diffusion-weighted imaging; EDAS = encephalodurosynangiosis; $E M S=$ encephalomyosynangiosis; $I C A=$ internal carotid artery; $i M M D=$ ischemic MMD; MCA = middle cerebral artery; MMD = moyamoya disease; $\mathrm{mRS}=$ modified Rankin Scale; $\mathrm{STA}=$ superficial temporal artery; TIA = transient ischemic attack. SUBMITTED November 5, 2019. ACCEPTED March 30, 2020.

INCLUDE WHEN CITING Published online June 12, 2020; DOI: 10.3171/2020.3.JNS192847. 
or hemorrhagic events is to establish blood flow augmentation to the affected hemisphere(s) by surgical revascularization, that is, an intracranial to extracranial (EC-IC) bypass procedure. This can be achieved by direct or indirect revascularization or by combined procedures. While it has been shown that direct bypass procedures are superior to conservative management in preventing future bleeding in hemorrhagic MMD, ${ }^{2}$ the best method of revascularization for ischemic MMD (iMMD) remains debatable. To date, no randomized trial has been performed to compare the efficacy of direct versus indirect procedures versus conservative management. Some authors believe that direct procedures are associated with higher morbidity rates than those following indirect procedures, and their reasoning is most commonly attributed to hyperperfusion syndrome and a higher perioperative stroke rate. ${ }^{3}$ On the other hand, the direct procedure is believed to provide a superior outcome in terms of protection from recurrent strokes.

We hypothesized that treatment selection-that is, direct versus indirect bypass procedure-is associated with surgical outcomes, including perioperative major stroke (within the 1st week) and extent of revascularization (on DSA) at 6 months postbypass. This study presents the results of an individualized selection strategy we utilized in the study period. The primary outcome measure was perioperative symptomatic stroke leading to a decline in the modified Rankin Scale (mRS) score at 6 months, and the secondary outcome measure was the degree of 6-month revascularization.

\section{Methods}

\section{Patient Demographic Data}

Appropriate institutional review board approval and patient consent were obtained for this study. Patients were identified retrospectively from a prospectively maintained database. Those treated for iMMD between March 2016 and June 2018 were included in the study, and all patients had been treated by the senior author. Patients with hemorrhagic presentation and patients receiving omental transposition as an indirect bypass graft were excluded. Demographic data were extracted from the patient charts.

\section{Preoperative Workup Data}

Our standard preoperative workup for patients with iMMD includes a DSA study not older than 3 months to the day of surgery and an acetazolamide (Diamox) challenge MRI study 1-2 weeks prior to surgery. The preoperative variables of previous stroke and/or TIA, Suzuki stage, degree of large vessel stenosis, and cerebrovascular reserve were registered. A previous stroke was defined as a localized neurological deficit with a corresponding T2 FLAIR-positive lesion. A TIA was defined as a temporary localized neurological deficit without a corresponding T2 FLAIR lesion. Suzuki stages were dichotomized as 1-3 (low grade) and 4-6 (high grade). ${ }^{4}$ Degree of large vessel stenosis was the degree of either ICA or middle cerebral artery (MCA) stenosis classified as mild, moderate, or severe stenosis or occlusion. Cerebrovascular reserve was assessed by intravenous acetazolamide challenge and categorized as preserved augmentation (> 10\% augmenta- tion from baseline), impaired or no augmentation, or steal phenomenon. The most impaired vascular territory was registered. Preoperative mRS score was extracted from the patient files.

\section{Operative Technique}

Mean arterial blood pressure was maintained at 5-10 $\mathrm{mm} \mathrm{Hg}$ above baseline during the procedure. For the graft, either the parietal or frontal branch of the superficial temporal artery (STA) was used in all but 1 case in which the occipital branch was used. The technique has been described elsewhere.,6 For both direct and indirect bypass, approximately 8-9 cm of graft was harvested and an approximately $6-\mathrm{cm}$-diameter craniotomy was fashioned. Direct bypass was performed as an STA-MCA end-to-side anastomosis using interrupted 10-0 sutures. Burst suppression was achieved with propofol during cross-clamping of the $\mathrm{M}_{4}$ segment. Bypass occlusion times generally ranged from 11 to 20 minutes. Anastomosis patency was assessed with indocyanine green in all cases. The remainder of the harvested STA with galeal cuff was laid on the cortical surface to promote indirect vessel growth. Indirect bypass included encephalodurosynangiosis (EDAS) in 42 hemispheres and encephalomyosynangiosis (EMS) in 1 hemisphere.

Intraoperatively, flow velocity and flow direction in the potential recipient $M_{4}$ vessel were registered with a Transonic Micro-Flowprobe (Transonic Systems Inc.). Flow away from the sylvian fissure was coded as anterograde flow and flow toward the sylvian fissure as retrograde flow.

\section{Selection Strategy}

Patients with at least 1 of these 4 criteria underwent indirect bypass: 1 ) mild to moderate ICA or $\mathrm{M}_{1}$ segment stenosis, 2) preserved cerebrovascular reserve, 3) intraoperative $\mathrm{M}_{4}$ segment anterograde flow (indicating flow away from the sylvian fissure) $\geq 8 \mathrm{ml} / \mathrm{min}$, and 4) the absence of frequent and severe TIAs, clinical strokes, or MRI evidence of chronic watershed infarction. The criteria for direct bypass were severe ICA or $\mathrm{M}_{1}$ segment stenosis or occlusion, impaired cerebrovascular reserve or steal phenomenon, intraoperative $\mathrm{M}_{4}$ segment retrograde flow or anterograde flow $<8 \mathrm{ml} / \mathrm{min}$, and the presence of frequent and severe TIAs or clinical strokes.

\section{Perioperative Period}

The perioperative period was defined as the first 7 days postoperatively. Our standard perioperative evaluation includes MRI during this period. A perioperative stroke was defined as a new symptomatic neurological deficit that decreased the patient's mRS score as compared to their preoperative baseline score (and persisting for at least 6 months) with a corresponding new diffusion-weighted imaging (DWI) lesion.

\section{Outcome}

Follow-up was preferably performed at 6 months postoperatively and included a clinical visit, MRI with acetazolamide challenge, and DSA. Any new symptomatic stroke and/or TIA during the perioperative period (defined 
as $\leq 7$ days postoperatively) was recorded. The postoperative mRS score was assessed at the 6-month follow-up appointment or, if not available, via structured telephone interview.

Postoperative revascularization was assessed on followup DSA as described previously. In brief, we scored revascularization as the proportion of the MCA territory that was revascularized from the bypass graft on lateral projection DSA, classifying it as $<1 / 3,1 / 3-2 / 3$, or $>2 / 3$ of the MCA territory (Fig. 1). The MCA territory was defined as the area not supplied by anterior cerebral artery collaterals anteriorly and posterior choroidal collaterals posteriorly.

\section{Statistical Analysis}

Distributions of the demographics of our cohort are described. Preoperative and postoperative clinical characteristics were compared between direct and indirect bypass groups. Median and interquartile ranges were reported for continuous variables. Pre- to postoperative improvement in $\mathrm{mRS}$ scores to 0 or 1 in each group was explored, with $\mathrm{p}$ values calculated by McNemar's chi-square test (only for those who had both pre- and postoperative scores). Comparisons of differences between direct and indirect bypass groups were conducted using the Mann-Whitney U-test for continuous variables and chi-square tests for categorical variables. To compare differences between direct and indirect groups with respect to primary and secondary outcomes (major stroke and revascularization score), multivariate regression analysis was performed, adjusting for age, preoperative stroke, and preoperative TIA. Logistic regression was used for major stroke, and linear regression was performed for revascularization score. We performed a complete case analysis, whereas surgeries with missing outcome and continuous covariates were excluded from the analysis and a missing category was created for categorical covariates that had missing values. Sensitivity analysis was conducted using generalized equation models to further account for the correlation of the surgeries within a patient. A p value $<0.05$ was considered statistically significant. All analysis was conducted using R Statistical Programming Language (version 3.4.3, R Foundation for Statistical Computing, https://www.R-project.org).

\section{Results \\ Demographics}

One hundred thirty-eight patients with iMMD affecting 195 hemispheres were included in the study. Twentytwo patients (15.9\%) were under 18 years of age (Table 1). Patients who did not present with either TIA or stroke were identified based on the presence of severe headaches, seizures, or syndrome (Down syndrome, microcephalic osteodysplastic primordial dwarfism type II [MOPDII]). One hundred thirty-three hemispheres $(68.2 \%)$ were revascularized via direct bypass and 62 hemispheres (31.8\%) via indirect bypass (Table 2). In 2 hemispheres a suitable donor was not found, and the hemisphere "crossed over" from a direct bypass to an indirect bypass. Ten hemispheres that did not fulfill the criteria for an indirect bypass received an indirect bypass. Five of these were in pediatric patients.

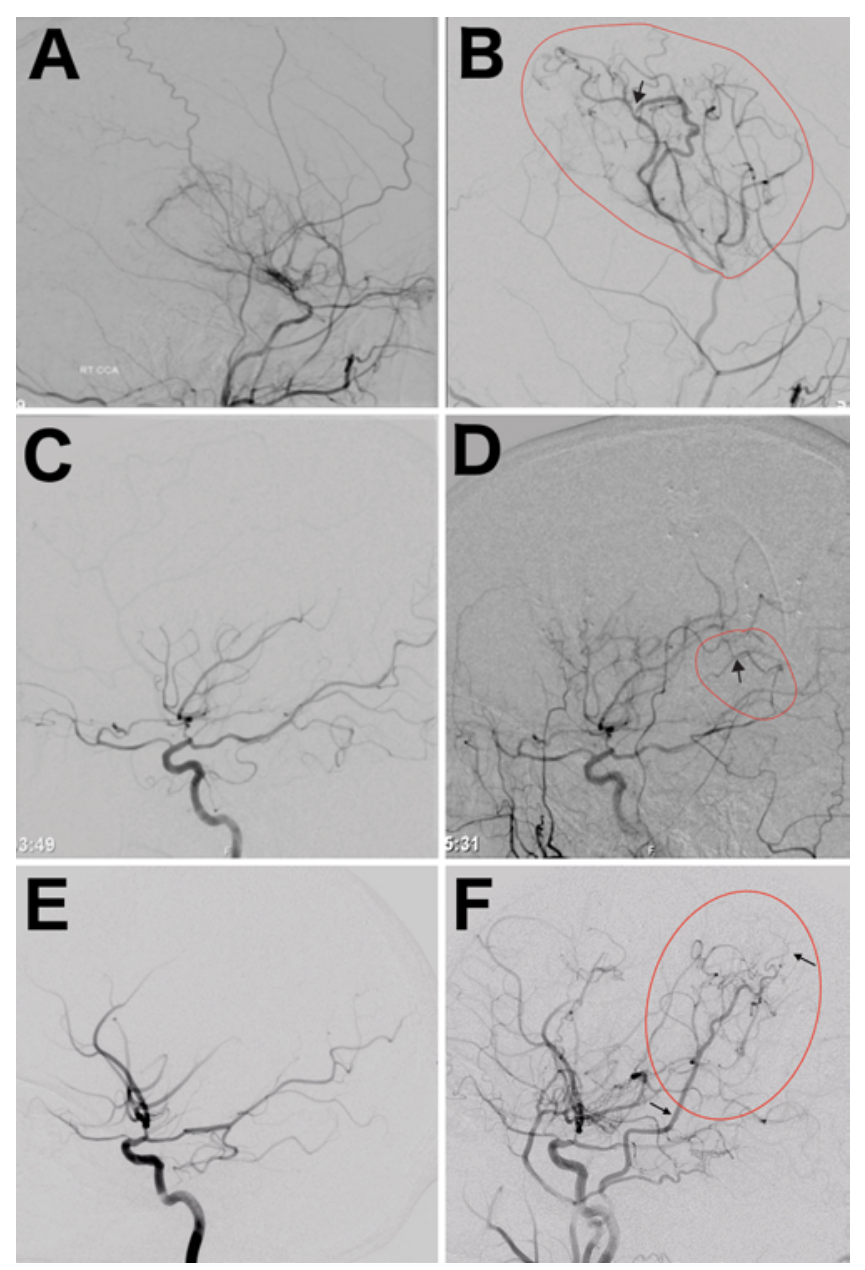

FIG. 1. Examples of radiological revascularization scores. A 21-yearold white female with bilateral MMD had previous FLAIR MRI-positive stroke and repeated TIAs. Preoperative acetazolamide challenge MRI demonstrated a steal phenomenon in the right MCA territory. Intraoperatively, she had retrograde $\mathrm{M}_{4}$ segment flow of $2 \mathrm{ml} / \mathrm{min}$. She was treated with a right STA-MCA bypass. A: Preoperative right common carotid artery (CCA) injection angiogram, lateral projection, demonstrating terminal ICA occlusion with moyamoya vessels. B: Postoperative right external carotid artery (ECA) injection angiogram, lateral projection, showing STA-MCA anastomosis (black arrow) and filling of $>2 / 3$ of the MCA territory (red outline). A 34-year-old Asian female with unilateral MMD had previous repeated TIAs but no FLAIR MRI-positive strokes. Preoperative acetazolamide challenge MRI demonstrated impaired augmentation in the left MCA territory. Intraoperatively, she had anterograde $\mathrm{M}_{4}$ segment flow of $5.5 \mathrm{ml} / \mathrm{min}$. She was treated with a left STA-MCA bypass. C: Preoperative left ICA injection angiogram, lateral projection, demonstrating severe terminal ICA stenosis with only a few moyamoya vessels. D: Postoperative ECA injection angiogram, lateral projection, showing the STA-MCA anastomosis (black arrow) and filling of $<1 / 3$ of the MCA territory (red outline). A 30 -year-old white female with bilateral MMD had previous repeated TIAs. Preoperative acetazolamide challenge MRI demonstrated impaired augmentation in the left MCA territory. Intraoperatively, she had anterograde $\mathrm{M}_{4}$ segment flow of $13.8 \mathrm{ml} /$ $\mathrm{min}$. She was treated with a left EDAS procedure. E: Preoperative left ICA injection angiogram, lateral projection, demonstrating moderate terminal ICA stenosis. F: Postoperative CCA injection angiogram, lateral projection, showing the indirect bypass from the STA (black arrows) with filling of $>2 / 3$ of the MCA territory (red outline). 
The severity of disease, cerebrovascular reserve, and intraoperative flow characteristics for each bypass modality are listed in Table 2. Patients in the direct bypass group had a significantly higher proportion of preoperative FLAIR-positive strokes, higher percentage of preoperative TIAs, higher degree of preoperative vessel stenosis, worse Suzuki stage, and greater percentage of retrograde $\mathrm{M}_{4}$ flow than the indirect bypass group. The direct bypass group tended toward a higher proportion of patients with a preoperative $\mathrm{mRS}$ score of 3 (Fig. 2A), but there was no significant difference in performance status between the two bypass groups. While blood flow augmentation after acetazolamide was 1 of the 4 possible inclusion criteria for indirect bypass, there was no difference in overall hemodynamic reserve between the treatment groups, although a higher percentage of patients in the indirect group (40.7\%) than in the direct group (34.4\%) had preserved reserve ( $\mathrm{p}$ $=0.70$ ).

\section{Outcome}

Clinical and radiological outcomes are listed in Table 3 and Fig. 2B. Follow-up angiograms were available for 134 hemispheres, and clinical follow-up was available for 132 hemispheres. Complete pre- and postoperative mRS scores were available for 110 patients. Postoperative MRI was not performed in 2 hemispheres, and these cases were excluded from the calculation of perioperative stroke.

The major perioperative stroke rate was $4.7 \%$ and $6.8 \%$ in the direct and indirect groups, respectively. After multivariate regression analysis adjusted for age, preoperative stroke, and preoperative TIA, no difference in the major stroke rate was detected between the groups ( $p=0.36$; Table 4). The transient neurological event (new neurological focal deficit with no new DWI infarct, resolving within 1 day to 2 weeks) was no different in the direct (19.8\%) and indirect groups $(24.2 \%$; $\mathrm{p}=0.62)$.

The median time from surgery to follow-up angiogram was 193 (IQR 66) days. A patent graft was confirmed in $96.6 \%$ and $97.8 \%$ of hemispheres in the direct and indirect groups, respectively. After adjusted multivariate regression analysis, the degree of revascularization was significantly higher in the direct bypass group than in the indirect $(\mathrm{p}=0.03$; Table 4).

The median time from surgery to clinical follow-up was 221 (IQR 84) days. Two cases in the direct group (2.2\%) had minor ischemic strokes during the follow-up period ( 1 week-6 months), not affecting the pre- to postoperative mRS score. Both cases had high-grade disease with low intraoperative flow velocities and a preoperative mRS score of 2 because of previous strokes. During the postoperative period, $28.8 \%$ and $20.5 \%$ of cases in the direct and indirect groups, respectively, experienced a TIA (Table 3). There was no difference in 6-month follow-up mRS scores between the treatment groups (Fig. 2B).

The proportion of patients with an mRS score of 0 or 1 whose preoperative and postoperative mRS scores had been recorded increased from $56 \%$ preoperatively to $62 \%$ postoperatively in the direct bypass group (Table 5). In the indirect group, the proportion of patients with an mRS score of 0 or 1 was $73 \%$ and $70 \%$ pre- and postoperatively, respectively. The improvement in pre- to postoperative
TABLE 1. Demographic data and disease laterality of 138 patients with iMMD

\begin{tabular}{cc}
\hline \multicolumn{1}{c}{ Variable } & \multicolumn{1}{c}{ Value } \\
\hline Sex, no. of patients (\%) \\
\hline F & $105(76.1)$ \\
\hline Median age in yrs (IQR) & $33(23.9)$ \\
\hline No. of adults (\%) & $37.5(21.5)$ \\
\hline No. of children: age <18 yrs (\%) & $116(84.1)$ \\
\hline Ethnicity, no. of patients (\%) & $22(15.9)$ \\
\hline Asian & $38(27.5)$ \\
\hline White & $71(51.4)$ \\
\hline Black & $9(6.5)$ \\
\hline Hispanic & $6(10.1)$ \\
\hline Other (including Polynesian) & $6(4.3)$ \\
\hline Laterality of disease & $96(49.2)$ \\
\hline Lt, no. of cases (\%) & $99(50.8)$ \\
\hline Rt, no. of cases (\%) & $45(32.6)$ \\
\hline Unilat, no. of patients (\%) & $93(67.4)$ \\
\hline Bilat, no. of patients (\%)
\end{tabular}

mRS scores in the direct group was not statistically significant $(\mathrm{p}=0.27)$. The pre- to postoperative "deterioration" in mRS scores in the indirect group was attributable to 1 patient and was not statistically significant $(\mathrm{p}=0.99)$.

Patients in both groups demonstrated improvements in their cerebrovascular reserve with a marked increase in the number of patients with preserved augmentation and decrease in steal phenomenon (Tables 2 and 3 ).

Four patients died during the 6-month postoperative period, and the mortality rate per operation for the entire cohort during this period was $3.0 \%$. One death was not related to any postoperative stroke, but to medical comorbidities.

A 46-year-old male died 4 days after a left-hemisphere indirect bypass (EDAS). He had had a preoperative mRS score of 4 because of a previous right-hemisphere stroke. He had undergone an uncomplicated right-hemisphere direct bypass 1 week prior to his indirect bypass. Although the patient had had a steal phenomenon and prior watershed infarcts in his left hemisphere, the infarcts were silent and his large vessel stenosis was found to be only moderate with a retrograde $\mathrm{M}_{4}$ flow of $12.4 \mathrm{ml} / \mathrm{min}$. For these reasons, an EDAS had been chosen. Immediately postoperatively, however, the patient developed an evolving left MCA territory infarct. With severe MCA strokes in both hemispheres, comfort care was instituted.

A 66-year-old female died 8 days after a left EDAS procedure. She had had bilateral MMD with previous strokes in the left hemisphere. Her preoperative mRS score had been 1 with severe large vessel occlusion $\left(M_{1}\right)$, steal phenomenon, and very low intraoperative flow of $0.9 \mathrm{ml} / \mathrm{min}$. She had awakened from anesthesia with expressive aphasia. MRI had demonstrated a left frontotemporal DWI lesion. This lesion had evolved over the course of the next days to ultimately involve the whole MCA territory as well 
TABLE 2. Preoperative characteristics in 138 patients with iMMD

\begin{tabular}{|c|c|c|c|c|}
\hline Variable & All & Direct Bypass Group & Indirect Bypass Group & $\mathrm{p}$ Value \\
\hline No. of hemispheres (\%) & 195 & $133(68.2)$ & $62(31.8)$ & \\
\hline Preop stroke, no. of cases (\%) & & & & 0.01568 \\
\hline No & $53(27.6)$ & $29(22.0)$ & $24(40.0)$ & \\
\hline Yes & $139(72.4)$ & $103(78.0)$ & $36(60.0)$ & \\
\hline Data missing & 3 & 1 & 2 & \\
\hline Preop TIA, no. of cases (\%) & & & & 0.0003 \\
\hline No & $60(31.3)$ & $30(22.7)$ & $30(50.0)$ & \\
\hline Yes & $132(68.8)$ & $102(77.3)$ & $30(50.0)$ & \\
\hline Data missing & 3 & 1 & 2 & \\
\hline Degree of preop vessel stenosis, no. of cases (\%) & & & & 0.0005 \\
\hline Mild & $5(2.6)$ & $0(0.0)$ & $5(8.1)$ & \\
\hline Moderate & $11(5.6)$ & $2(1.5)$ & $9(14.5)$ & \\
\hline Severe & $45(23.1)$ & $16(12.0)$ & $29(46.8)$ & \\
\hline Occlusion & $134(68.7)$ & $115(86.5)$ & $19(30.6)$ & \\
\hline Suzuki stage, no. of cases (\%) & & & & 0.0026 \\
\hline 1-3 (low grade) & $109(56.5)$ & $65(48.9)$ & $44(73.3)$ & \\
\hline 4-6 (high grade) & $84(43.5)$ & $68(51.1)$ & $16(26.7)$ & \\
\hline Data missing & 2 & 0 & 2 & \\
\hline Preop hemodynamic reserve, no. of cases (\%) & & & & 0.69517 \\
\hline Augmentation & $65(36.3)$ & $43(34.4)$ & $22(40.7)$ & \\
\hline Impaired or no augmentation & $77(43.0)$ & $56(44.8)$ & $21(38.9)$ & \\
\hline Steal phenomenon & $37(20.7)$ & $26(20.8)$ & $11(20.4)$ & \\
\hline Data missing & 16 & 8 & 8 & \\
\hline Preop mRS score, no. of cases (\%) & & & & 0.30535 \\
\hline 0 & $6(4.8)$ & $4(4.5)$ & $2(5.4)$ & \\
\hline 1 & $65(52.0)$ & $41(46.6)$ & $24(64.9)$ & \\
\hline 2 & $43(34.4)$ & $35(39.8)$ & $8(21.6)$ & \\
\hline 3 & $8(6.4)$ & $6(6.8)$ & $2(5.4)$ & \\
\hline 4 & $3(2.4)$ & $2(2.3)$ & $1(2.7)$ & \\
\hline Data missing & 70 & 45 & 25 & \\
\hline Preop $\mathrm{M}_{4}$ flow direction, no. of cases (\%) & & & & 0.0010 \\
\hline Anterograde & $136(72.3)$ & $85(64.9)$ & $51(89.5)$ & \\
\hline Retrograde & $52(27.7)$ & $46(35.1)$ & $6(10.5)$ & \\
\hline Data missing & 7 & 2 & 5 & \\
\hline Median $\mathrm{M}_{4}$ flow in $\mathrm{ml} / \mathrm{min}(\mathrm{IQR})$ & $4.0(2.1,6.9)$ & $3.7(2.0,5.5)$ & $7.1(3.0,12.8)$ & 0.0000 \\
\hline
\end{tabular}

as the posterior cerebral artery territory, developing into a malignant MCA infarction. A decompressive hemicraniectomy had been performed but did not improve the patient's clinical status, and active treatment was withdrawn.

A 40-year-old female died after a left EDAS procedure. This patient had presented with a complex medical history of acute lymphatic leukemia at 18 months of age, which had been treated with both chemotherapy and radiation. Because of this she had developed a cardiomyopathy that subsequently required a heart transplant. She had also had kidney failure and was on dialysis. She had had bilateral MMD with the left side symptomatic. Her preoperative mRS score had been 3 with severe large vessel stenosis. Preoperative assessment of cerebrovascular reserve had not been performed in this patient. Given her complex comorbidity, an indirect bypass had been chosen. The patient had seizure activity postoperatively but did not suffer any significant new postoperative strokes. However, she had a prolonged ICU stay complicated by respiratory and cardiac failure and eventually became septic. She died 12 days after surgery. As death was attributable to her complex medical history and she had not had a postoperative stroke, the case was not coded as a major stroke.

The fourth patient, a 46-year-old female, had presented with previous strokes, high-grade stenosis, impaired reserve, and an $\mathrm{mRS}$ score of 2 . She had had low perioperative anterograde $\mathrm{M}_{4}$ flow. An uneventful direct revascularization procedure had been performed, and the patient woke up immediately. On postoperative day 2 , however, she developed multiple progressive ischemic lesions in 
Nielsen et al.
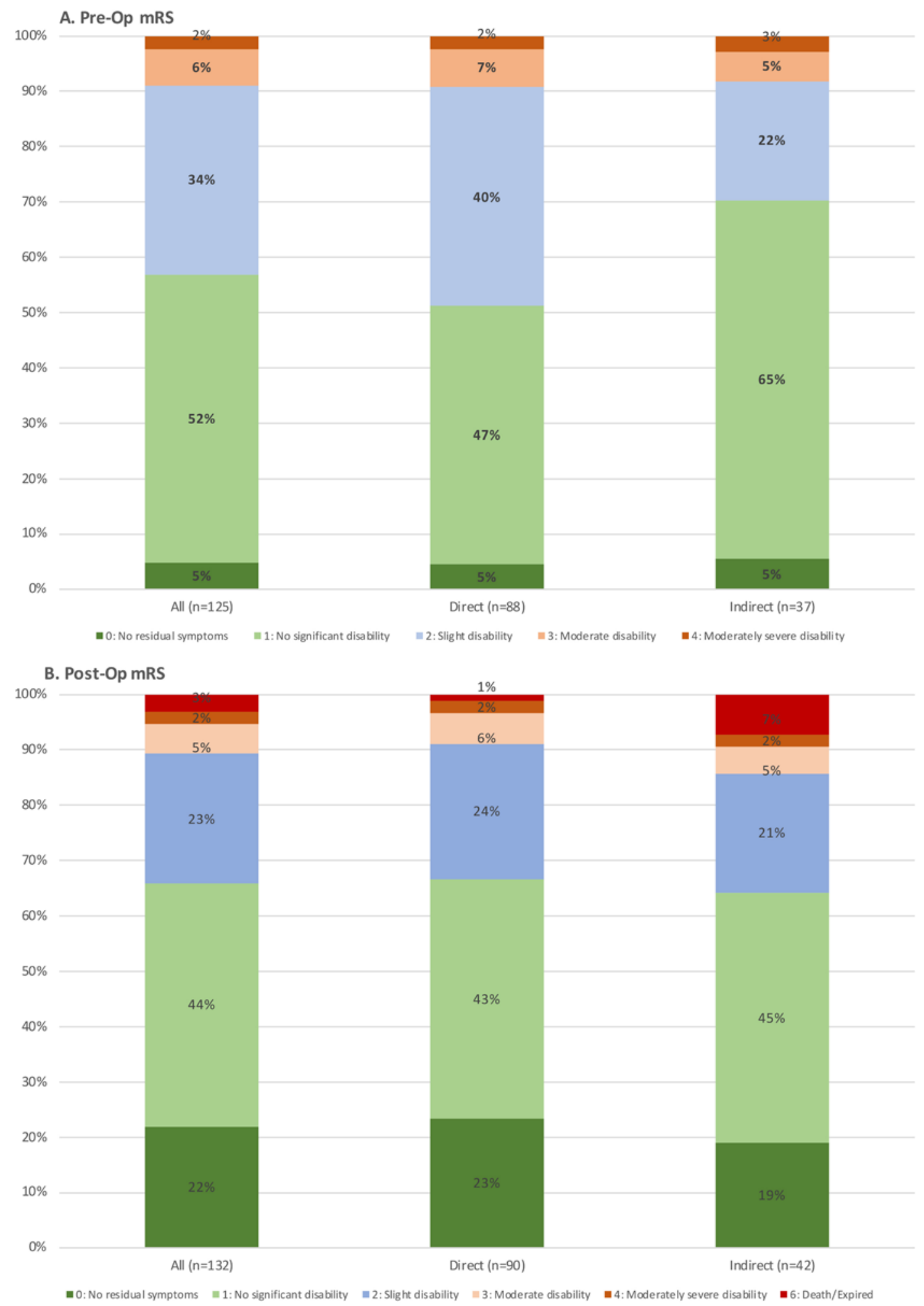

FIG. 2. Distribution of mRS scores per operated hemisphere preoperatively (A) and at the 6-month follow-up (B). 
TABLE 3. Peri- and postoperative outcome variables

\begin{tabular}{|c|c|c|c|c|}
\hline Variable & All & Direct Bypass Group & Indirect Bypass Group & $\mathrm{p}$ Value \\
\hline No. of hemispheres & 195 & 133 & 62 & \\
\hline Periop seizure, no. of cases (\%) & & & & 0.58927 \\
\hline No & $170(91.4)$ & $114(90.5)$ & $56(93.3)$ & \\
\hline Yes & $16(8.6)$ & $12(9.5)$ & $4(6.7)$ & \\
\hline Data missing & 9 & 7 & 2 & \\
\hline Periop TNE, no. of cases (\%) & & & & 0.61647 \\
\hline No & $152(78.8)$ & $105(80.2)$ & $47(75.8)$ & \\
\hline Yes & $41(21.2)$ & $26(19.8)$ & $15(24.2)$ & \\
\hline Data missing & 2 & 2 & 0 & \\
\hline Major periop stroke, no. of cases (\%) & & & & 0.72794 \\
\hline Yes & $10(5.4)$ & $6(4.7)$ & $4(6.8)$ & \\
\hline No & $176(94.6)$ & $121(95.3)$ & $55(93.2)$ & \\
\hline Data missing & 9 & 6 & 3 & \\
\hline Revascularization, no. of cases (\%) & & & & 0.01052 \\
\hline$<1 / 3$ of $\mathrm{MCA}$ & $56(41.5)$ & $29(32.6)$ & $27(58.7)$ & \\
\hline $1 / 3-2 / 3$ of MCA & $40(29.6)$ & $32(36.0)$ & $8(17.4)$ & \\
\hline$>2 / 3$ of $M C A$ & $39(28.9)$ & $28(31.5)$ & $11(23.9)$ & \\
\hline Data missing & 60 & 44 & 16 & \\
\hline Patent graft, no. of cases (\%) & & & & 0.81109 \\
\hline No & $4(3.0)$ & $3(3.4)$ & $1(2.2)$ & \\
\hline Yes & $130(97.0)$ & $86(96.6)$ & $44(97.8)$ & \\
\hline Data missing & 61 & 44 & 17 & \\
\hline Postop TIA, no. of cases (\%) & & & & 0.42626 \\
\hline No & $92(74.2)$ & $57(71.3)$ & $35(79.5)$ & \\
\hline Yes & $32(25.8)$ & $23(28.8)$ & $9(20.5)$ & \\
\hline Data missing & 71 & 53 & 18 & \\
\hline \multicolumn{5}{|l|}{ Postop stroke, no. of cases (\%) } \\
\hline No & $130(98.5)$ & $88(97.8)$ & $42(100)$ & 0.99 \\
\hline Yes & $2(1.5)$ & $2(2.2)$ & $0(0)$ & \\
\hline Data missing & 63 & 43 & 20 & \\
\hline Postop reserve, no. of cases (\%) & & & & 0.04848 \\
\hline Augmentation & $78(72.9)$ & $45(68.2)$ & $33(80.5)$ & \\
\hline Impaired or no augmentation & $25(23.4)$ & $20(30.3)$ & $5(12.2)$ & \\
\hline Steal phenomenon & $4(3.7)$ & $1(1.5)$ & $3(7.3)$ & \\
\hline Data missing & 88 & 67 & 21 & \\
\hline Postop mRS score, no. of cases (\%) & & & & 0.56172 \\
\hline 0 & $29(22.0)$ & $21(23.3)$ & $8(19.0)$ & \\
\hline 1 & $58(43.9)$ & $39(43.3)$ & $19(45.2)$ & \\
\hline 2 & $31(23.5)$ & $22(24.4)$ & $9(21.4)$ & \\
\hline 3 & $7(5.3)$ & $5(5.6)$ & $2(4.8)$ & \\
\hline 4 & $3(2.3)$ & $2(2.2)$ & $1(2.4)$ & \\
\hline 6 & $4(3.0)$ & $1(1.1)$ & $3(7.1)$ & \\
\hline Data missing & 63 & 43 & 20 & \\
\hline
\end{tabular}

TNE = transient neurological event.

Perioperative period was defined as days 1-7 after surgery. Postoperative period was defined as day 7 after surgery until clinical follow-up. 
TABLE 4. Regression analysis comparing differences between direct and indirect bypass groups

\begin{tabular}{|c|c|c|c|c|c|c|c|}
\hline \multirow[b]{2}{*}{ Outcome } & \multirow{2}{*}{$\begin{array}{c}\text { No. of } \\
\text { Observations Used }\end{array}$} & \multicolumn{3}{|c|}{ Unadjusted Results } & \multicolumn{3}{|c|}{ Adjusted Results } \\
\hline & & OR or Estimate & $95 \% \mathrm{Cl}$ & $p$ Value & OR or Estimate & $95 \% \mathrm{Cl}$ & $p$ Value \\
\hline Major stroke* & 186 & 1.47 & $0.36,5.34$ & 0.56504 & 2.07 & $0.44,9.72$ & 0.35722 \\
\hline Revascularization score $†$ & 135 & 0.34 & $0.04,0.63$ & 0.02541 & 0.34 & $0.03,0.66$ & 0.03066 \\
\hline mRS score $†$ & 132 & 0.30 & $-0.15,0.76$ & 0.18988 & 0.27 & $-0.17,0.72$ & 0.22788 \\
\hline
\end{tabular}

The reference group was the direct bypass group. Analyses were adjusted for age, preoperative stroke, and preoperative TIA.

* Logistic regressions were performed and odds ratios are reported.

$\dagger$ Linear regressions were performed.

both hemispheres, ultimately leading to extensive bilateral infarction and death.

One patient was lost to follow-up, but she died of an unknown cause 13 months after her surgery.

\section{Pediatric Cohort}

A subgroup analysis with respect to perioperative major stroke rate, postoperative mRS score, and revascularization in the pediatric cohort is shown in Supplementary Table 1. No significant effect of bypass type on any of these variables was found. However, it is noteworthy that there was better revascularization from a direct bypass ( $\mathrm{p}$ $=0.079$ ).

\section{Discussion}

In the present study, we tested the hypothesis that assignment to either direct or indirect bypass for iMMD based on hemodynamic, clinical, and radiological parameters would result in different rates of perioperative major stroke and 6-month extent of revascularization. One assumption was that the perioperative stroke rate after indirect bypass would be lower than that after direct bypass.

\section{Selection Strategy}

Various scoring systems based on clinical and quantitative variables have been proposed to describe the severity of disease, to guide decision-making, and to predict perioperative complications. ${ }^{8-10}$ These scoring systems incorporate clinical features such as ischemic symptoms,

TABLE 5. Distribution of $\mathrm{mRS}$ scores in the pre- and postoperative periods

\begin{tabular}{lcc}
\hline Group & mRS Score 0-1 & $m R S$ Score $>1$ \\
\hline Direct bypass $(n=77)$ & $43(56 \%)$ & $34(44 \%)$ \\
\hline Preop & $48(62 \%)$ & $29(38 \%)$ \\
\hline Postop & \multicolumn{3}{c}{0.2673} \\
\hline p value & \multicolumn{3}{c}{$9(27 \%)$} \\
\hline Indirect bypass $(n=33)$ & $24(73 \%)$ & $10(30 \%)$ \\
\hline Preop & $23(70 \%)$ & \\
\hline Postop & \multicolumn{3}{c}{0.99} \\
\hline p value
\end{tabular}

$n=$ number of patients with both pre- and postoperative scores.

A $p$ value for the difference between periods within each group was calculated by McNemar's chi-square test. hemodynamic features, and Suzuki staging. One challenge in setting up a scoring system is how to weigh the different variables against each other in terms of perioperative stroke risk. In fact, it is unknown whether a preoperative blood flow steal phenomenon carries a higher risk of perioperative stroke than preoperative arterial high-grade stenosis/occlusion or low intraoperative recipient $\mathrm{M}_{4}$ arterial flow. Further, a scoring system should not only consider the perioperative stroke risk, but also balance this risk against the subsequent protection from future strokes.

It has been shown that higher-grade moyamoya characterized by a higher degree of large vessel stenosis, previous and repeated strokes, and poor cerebrovascular reserve has a higher postoperative risk of ischemic complications..$^{10-13}$ Moreover, a recent stroke seems to be an especially significant independent risk factor for perioperative stroke..$^{10-13}$ In line with these results, Funaki and colleagues reported a $4.5 \%$ risk of a new perioperative DWI lesion in a socalled stable moyamoya population, but a $33.3 \%$ risk in an unstable group characterized by rapid disease progression and repeated strokes. ${ }^{14}$ Further, our group recently demonstrated an increased perioperative stroke risk for high-grade patients based on the degree of vessel stenosis/ occlusion, cerebrovascular reserve, and preoperative presence of ischemic lesions..$^{10}$

We have previously shown that local increased flow in the recipient vessel is associated with clinical improvement. ${ }^{15}$ Hence, we considered intraoperative prebypass flow direction and flow rate in our selection procedure for direct versus indirect bypass. Furthermore, extremely low or reversed $\mathrm{M}_{4}$ flow prebypass may also be a predictor of perioperative stroke following direct bypass, as very high flow augmentation is associated with perioperative stroke $\mathrm{e}^{15}$ possibly because a direct bypass with higher flow imposes a sudden change in hemodynamics leading to competing flow in those patients with pre-anastomotic low or retrograde flow. ${ }^{16}$

Consistent allocation to either direct or indirect bypass from the predefined variables was confirmed by the fact that patients in the direct bypass group had significantly higher proportions of preoperative FLAIR MRI-positive stroke, TIA, and terminal ICA stenosis; higher Suzuki stages; lower $\mathrm{M}_{4}$ flows; and greater percentage of retrograde $\mathrm{M}_{4}$ flow than the indirect bypass group. However, there was no significant difference in preoperative hemodynamic reserve between the bypass groups (Table 2). This finding reflects the fact that patients did not have to fulfill all criteria for inclusion in the direct bypass group and that the other criteria (preoperative ischemic symp- 
toms, ICA stenosis, Suzuki stage, $\mathrm{M}_{4}$ flow) were weighted higher in our selection strategy. This implies that patients with impaired hemodynamic reserve may have been allocated to indirect bypass if the patient did not have preoperative FLAIR strokes but did have lower-grade occlusive disease and higher $\mathrm{M}_{4}$ flow, and vice versa.

Impaired hemodynamic reserve is considered an indicator of high-grade disease..$^{10}$ Conversely, Funaki and colleagues did not find preoperative compromised hemodynamic reserve to be associated with postoperative DWI-detected lesions. ${ }^{14}$ Whether impaired hemodynamic reserve increased the stroke rate in our indirect bypass group is unknown.

\section{Perioperative Stroke Rate}

The perioperative stroke rate (within 7 days of surgery) was not different between the direct $(4.7 \%)$ and indirect (6.8\%) groups. These perioperative stroke rates are comparable to those reported in our prior study ${ }^{17}$ and by other authors..$^{18,19}$ A recent large, retrospective, single-center study of 610 hemispheres reported an equal postoperative ischemic complication rate between $4 \%$ and 5\% among direct, indirect, and combined procedures. ${ }^{20}$ Our previous study of 450 bypass procedures for MMD revealed a perioperative major stroke rate of $1.7 \%$ and mortality rate of $0.7 \%$ per treated hemisphere. ${ }^{17}$ In that study cohort, 95\% of adults and $76 \%$ of pediatric patients were treated with a direct revascularization technique. The fact that neither the perioperative major stroke rate nor the perioperative transient neurological event rate differed between direct and indirect groups suggests that these complications are not simply related to competing flows between the direct graft and the native circulations, as has been previously proposed.

Our center has treated more than 1150 patients with MMD and performed greater than 1780 EC-IC bypasses for this disease. In the last decade, the anastomosis technique has remained basically unchanged ${ }^{21}$ with a recipient $\mathrm{M}_{4}$ occlusion time under 20 minutes in general. Further, peri- and postoperative care have remained unchanged.

The cohort in the present study had rather high proportions of preoperative strokes (72.4\%) and TIAs (68.8\%) compared to others with a reported presentation ischemic stroke rate between $33.6 \%$ and $48.3 \% .^{20,22}$ The current preoperative stroke rate was also higher than that in our previously presented cohort. ${ }^{17}$ Further, there was a high proportion of cases with impaired hemodynamic reserve (63.7\%), with $20.7 \%$ of the cases demonstrating steal phenomenon (Table 2). While we routinely avoid revascularization within 2 weeks of an acute stroke, a few patients had frequent repeated strokes and were revascularized within 2 weeks from a stroke. All these factors may have contributed to the observed stroke rate in both the direct and indirect bypass groups.

A number of patients in our study did not present with either TIA or stroke. Cho and colleagues followed 241 conservatively managed moyamoya patients for a mean of 82.5 months. ${ }^{23}$ They found that the annual stroke rate was $4.5 \%$ in an adult moyamoya population with stable disease (defined by stable hemodynamic features on SPECT). This stroke rate also applied to asymptomatic moyamoya patients. These findings indicate that even asymptomatic and presumably stable moyamoya patients carry a substantial lifetime risk of stroke, but also a risk of perioperative stroke.

\section{Clinical Outcome}

We observed an increase in the proportion of patients improving to an mRS score of 0 or 1 from $56 \%$ to $62 \%$ in the direct bypass group, although the difference in scores at the two time points was not significant (Fig. 2 and Table 5). In this group, $77.3 \%$ of cases had experienced preoperative TIAs and $78.0 \%$ had experienced preoperative stroke (Table 2). This proportion decreased postoperatively to $28.8 \%$ and $2.2 \%$ for TIA and stroke, respectively (Table 3). Hence, the improvement in mRS scores is most likely attributable to the decrease or absence of TIA and stroke. Other factors contributing to improvement in $\mathrm{mRS}$ scores are most likely decreased fatigue and improvement in headache, whereas neurocognitive improvement is not expected..$^{24}$

The same improvement in the percentage of patients attaining mRS scores 0 and 1 was not observed in the indirect bypass group (Table 5 ). In this group, $50 \%$ and $60 \%$ experienced preoperative TIA or stroke, respectively. These numbers decreased to $20.5 \%$ and $0 \%$, respectively, in the postoperative period ( 1 week- 6 months). This finding suggests that symptoms other than TIA and stroke impact patients' lives (e.g., fatigue and headache) and that the flow augmentation provided by a direct bypass may be superior in relieving these symptoms.

\section{Radiological Outcome}

Radiological revascularization may be considered as a surrogate measure for outcome in terms of symptom improvement and protection from future strokes. We recorded the angiographic neoangiogenesis at 6 months postoperatively, as full angiogenesis can be expected within the first 6 months after revascularization. ${ }^{25}$ Few studies have compared the angiographic outcome after revascularization from direct and indirect bypass. One small study has demonstrated equal proportions of Matsushima grade A and $\mathrm{B}$ revascularizations in adults after either direct or indirect bypass, ${ }^{26}$ but only 4 of 36 procedures included a direct bypass. Another study has shown significantly better revascularization from direct or combined procedures than with indirect bypass alone. ${ }^{27}$ In an another small study of 20 procedures, a significantly higher proportion of good angiographic outcomes after direct revascularization was reported. ${ }^{28}$ In our overall cohort, direct bypass provided superior revascularization compared to that with indirect bypass (Table 4), and the same trend was observed in the pediatric cohort alone (Supplementary Table 1). On the other hand, both bypass groups experienced marked improvement in cerebrovascular reserve (Tables 2 and 3). In fact, postoperative cerebrovascular reserve was significantly better in the indirect group than in the direct group, suggesting that the radiological revascularization score is not necessarily correlated with the postoperative cerebrovascular reserve and that an indirect bypass can provide sufficient revascularization to improve cerebrovascular reserve. This is not surprising and most likely reflects the 
fact that the degree of revascularization depends on the requirement for flow augmentation. However, this conclusion should be drawn with caution. Cerebrovascular reserve was one of our selection criteria and therefore subject to selection bias. Although the difference between the two groups was not statistically significant, the direct bypass group showed worse preoperative hemodynamic reserve than the indirect group. Accordingly, whether this finding of improved hemodynamic reserve after bypass translates into a superior clinical outcome in terms of protection from future strokes cannot be determined.

\section{Pediatric Considerations}

Traditionally, the pediatric population is believed to have a higher potential for successful revascularization from an indirect bypass than a direct bypass. ${ }^{29-31}$ On the contrary, others have concluded that the combined STAMCA direct bypass and EMS provides a significantly higher revascularization rate and improvement in clinical outcome than EDAS in a pediatric population. ${ }^{7}$ We could not demonstrate any statistically significant difference in the stroke rate between the groups. However, there was a trend toward better revascularization from a direct bypass.

\section{Overall Implications}

In a series of 140 moyamoya-affected hemispheres, Abla and colleagues found that direct and indirect techniques were equally effective in preventing future moyamoya-associated strokes, though direct procedures were superior in achieving symptom improvement in the adult population. ${ }^{22}$ Deng and colleagues found that direct procedures were more effective in preventing recurrent ischemic stroke than indirect procedures, although there was no difference in functional outcome. ${ }^{32}$ In line with these results, three meta-analyses demonstrated that indirect bypass was less effective in stroke reduction than direct bypass, with no significant difference in perioperative complications between the two modalities. ${ }^{33-35}$ On the contrary, in a recent comprehensive review of long-term follow-up after revascularization for MMD, Macyszyn and colleagues showed that indirect procedures were significantly superior to direct procedures in providing quality-adjusted life years. ${ }^{36}$ Also, a recent single-center study comparing direct and indirect bypass recommended the latter, suggesting that indirect bypass is sufficient for adult MMD, taking into consideration the complication rate, operating time, length of hospital stay, long-term revascularization, and clinical outcome. ${ }^{37}$ The divergent results from the different studies probably reflect the heterogeneity in patient characteristics and patient selection between studies, as well as varied lengths of follow-up.

Our rationale for preselecting patients to undergo either direct or indirect bypass based on clinical and hemodynamic factors was to reduce the heterogeneity of MMD patients by identifying those who would benefit from an indirect bypass alone, thereby decreasing the perioperative stroke rate. We did not accomplish this goal. The stroke rate was comparable between the groups, direct bypass provided superior angiographic revascularization, and indirect bypass provided superior improvement in hemodynamic reserve. Nonetheless, an improvement in the $\mathrm{mRS}$ score was observed in the direct bypass group but not the indirect bypass group, most likely due to relief from strokes and TIAs as well as the concomitant symptoms frequently associated with MMD. Given our results, we would recommend a direct bypass procedure for the treatment of iMMD.

\section{Study Limitations}

Although this study was prospectively planned to select patients for either direct or indirect bypass on the basis of certain criteria, it was not a randomized study, and preoperative and follow-up data were incomplete for some variables (Tables 2 and 3). Because of comorbidities, a few patients were assigned to indirect bypass despite hemodynamic features that would have allocated them to the direct group, thus introducing a selection bias. This might have rendered them more prone to ischemic complications, thereby increasing the perioperative stroke rate in the indirect group. However, the regression analysis adjusted for preoperative strokes and TIAs and therefore, in part, accounts for this bias. Also, the follow-up period was relatively short. While 6 months' follow-up is sufficient for assessing radiological revascularization and hemodynamic reserve, it is insufficient to assess protection from future strokes. Improved angiographic revascularization may be a surrogate measure for better protection from ischemic stroke, but whether this translates into clinical reality cannot be confirmed based on our current data. Prospective follow-up of the cohort will demonstrate whether the superior angiographic revascularization in the direct bypass group translates into superior clinical outcomes.

\section{Conclusions}

An individualized selection strategy to either a direct or indirect bypass procedure for iMMD did not decrease the perioperative stroke rate, which was comparable between the two procedures. Direct bypass provided superior angiographic revascularization and improvement in the $\mathrm{mRS}$ score. We recommend this procedure as preferable for the treatment of adult iMMD. Long-term follow-up is necessary to demonstrate whether superior angiographic revascularization translates into increased protection from future strokes.

\section{Acknowledgments}

This study was supported in part by Russell and Elizabeth Siegelman, Bernard and Ronni Lacroute, the William Randolph Hearst Foundation, and the Reddy Lee Family Fund (G.K.S.).

We thank Jeanne $\mathrm{Gu}$ for database maintenance and data extraction and Christine Plant for editorial assistance with the manuscript.

\section{References}

1. Suzuki J, Takaku A. Cerebrovascular "moyamoya" disease. Disease showing abnormal net-like vessels in base of brain. Arch Neurol. 1969;20(3):288-299.

2. Miyamoto S, Yoshimoto T, Hashimoto N, et al. Effects of extracranial-intracranial bypass for patients with hemorrhagic moyamoya disease: results of the Japan Adult Moyamoya Trial. Stroke. 2014;45(5):1415-1421. 
3. Yu J, Shi L, Guo Y, et al. Progress on complications of direct bypass for moyamoya disease. Int J Med Sci. 2016;13(8):578587.

4. Wang L, Qian C, Yu X, et al. Indirect bypass surgery may be more beneficial for symptomatic patients with moyamoya disease at early Suzuki stage. World Neurosurg. 2016;95:304308.

5. Chang SD, Steinberg GK. Superficial temporal artery to middle cerebral artery anastomosis. Tech Neurosurg. 2000;6(2):86-100.

6. Gooderham P, Steinberg GK. Intracranial-extracranial bypass surgery for moyamoya disease. In: Spetzler MKY, Nakaji P, eds. Neurovascular Surgery. Thieme; 2015:1156-1171.

7. Matsushima T, Inoue T, Suzuki SO, et al. Surgical treatment of moyamoya disease in pediatric patients - comparison between the results of indirect and direct revascularization procedures. Neurosurgery. 1992;31(3):401-405.

8. Ladner TR, Donahue MJ, Arteaga DF, et al. Prior Infarcts, Reactivity, and Angiography in Moyamoya Disease (PIRAMD): a scoring system for moyamoya severity based on multimodal hemodynamic imaging. J Neurosurg. 2017;126(2):495-503.

9. Kashiwazaki D, Akioka N, Kuwayama N, et al. Berlin grading system can stratify the onset and predict perioperative complications in adult moyamoya disease. Neurosurgery. 2017;81(6):986-991.

10. Teo M, Furtado S, Kaneko OF, et al. Validation and application for the Berlin grading system of moyamoya disease in adult patients. Neurosurgery. 2020;86(2):203-212.

11. Czabanka M, Boschi A, Acker G, et al. Grading of moyamoya disease allows stratification for postoperative ischemia in bilateral revascularization surgery. Acta Neurochir (Wien). 2016;158(10):1895-1900.

12. Hyun SJ, Kim JS, Hong SC. Prognostic factors associated with perioperative ischemic complications in adult-onset moyamoya disease. Acta Neurochir (Wien). 2010;152(7):1181-1188.

13. Kim SH, Choi JU, Yang KH, et al. Risk factors for postoperative ischemic complications in patients with moyamoya disease. J Neurosurg. 2005;103(5)(suppl):433-438.

14. Funaki T, Takahashi JC, Takagi Y, et al. Unstable moyamoya disease: clinical features and impact on perioperative ischemic complications. J Neurosurg. 2015;122(2):400-407.

15. Lee M, Guzman R, Bell-Stephens T, Steinberg GK. Intraoperative blood flow analysis of direct revascularization procedures in patients with moyamoya disease. J Cereb Blood Flow Metab. 2011;31(1):262-274.

16. Hayashi T, Shirane R, Fujimura M, Tominaga T. Postoperative neurological deterioration in pediatric moyamoya disease: watershed shift and hyperperfusion. J Neurosurg Pediatr. 2010;6(1):73-81.

17. Guzman R, Lee M, Achrol A, et al. Clinical outcome after 450 revascularization procedures for moyamoya disease. Clinical article. J Neurosurg. 2009;111(5):927-935.

18. Park W, Ahn JS, Lee HS, et al. Risk factors for newly developed cerebral infarction after surgical revascularization for adults with moyamoya disease. World Neurosurg. 2016;92:65-73.

19. Kazumata K, Ito M, Tokairin K, et al. The frequency of postoperative stroke in moyamoya disease following combined revascularization: a single-university series and systematic review. J Neurosurg. 2014;121(2):432-440.

20. Zhao M, Deng X, Zhang D, et al. Risk factors for and outcomes of postoperative complications in adult patients with moyamoya disease. J Neurosurg. 2019;130(2):531-542.

21. Charbel FT, Meglio G, Amin-Hanjani S. Superficial temporal artery-to-middle cerebral artery bypass. Neurosurgery. 2005;56(1)(suppl):186-190.

22. Abla AA, Gandhoke G, Clark JC, et al. Surgical outcomes for moyamoya angiopathy at barrow neurological institute with comparison of adult indirect encephaloduroarteriosynangiosis bypass, adult direct superficial temporal artery-to-middle cerebral artery bypass, and pediatric bypass: 154 revascularization surgeries in 140 affected hemispheres. Neurosurgery. 2013;73(3):430-439.

23. Cho WS, Chung YS, Kim JE, et al. The natural clinical course of hemodynamically stable adult moyamoya disease. $J$ Neurosurg. 2015;122(1):82-89.

24. Zeifert PD, Karzmark P, Bell-Stephens TE, et al. Neurocognitive performance after cerebral revascularization in adult moyamoya disease. Stroke. 2017;48(6):1514-1517.

25. Zhao Y, Lu J, Zhang Q, et al. Time course of neoangiogenesis after indirect bypass surgery for moyamoya disease: comparison of short-term and long-term follow-up angiography. Clin Neuroradiol. 2020;30(1):91-99.

26. Sahoo SS, Suri A, Bansal S, et al. Outcome of revascularization in moyamoya disease: evaluation of a new angiographic scoring system. Asian J Neurosurg. 2015;10(4):252-259.

27. Bang JS, Kwon OK, Kim JE, et al. Quantitative angiographic comparison with the OSIRIS program between the direct and indirect revascularization modalities in adult moyamoya disease. Neurosurgery. 2012;70(3):625-633.

28. Arias EJ, Dunn GP, Washington CW, et al. Surgical revascularization in North American adults with moyamoya phenomenon: long-term angiographic follow-up. J Stroke Cerebrovasc Dis. 2015;24(7):1597-1608.

29. Fujita K, Tamaki N, Matsumoto S. Surgical treatment of moyamoya disease in children: which is more effective procedure, EDAS or EMS? Childs Nerv Syst. 1986;2(3):134-138.

30. Manceau E, Giroud M, Dumas R. Moyamoya disease in children. A review of the clinical and radiological features and current treatment. Childs Nerv Syst. 1997;13(11-12):595-600.

31. Matsushima T, Fujiwara S, Nagata S, et al. Surgical treatment for paediatric patients with moyamoya disease by indirect revascularization procedures (EDAS, EMS, EMAS). Acta Neurochir (Wien). 1989;98(3-4):135-140.

32. Deng X, Gao F, Zhang D, et al. Direct versus indirect bypasses for adult ischemic-type moyamoya disease: a propensity score-matched analysis. J Neurosurg. 2018;128(6):1785-1791.

33. Qian C, Yu X, Li J, et al. The efficacy of surgical treatment for the secondary prevention of stroke in symptomatic moyamoya disease: a meta-analysis. Medicine (Baltimore). 2015;94(49):e2218.

34. Jeon JP, Kim JE, Cho WS, et al. Meta-analysis of the surgical outcomes of symptomatic moyamoya disease in adults. $J$ Neurosurg. 2018;128(3):793-799.

35. Sun H, Wilson C, Ozpinar A, et al. Perioperative complications and long-term outcomes after bypasses in adults with moyamoya disease: a systematic review and meta-analysis. World Neurosurg. 2016;92:179-188.

36. Macyszyn L, Attiah M, Ma TS, et al. Direct versus indirect revascularization procedures for moyamoya disease: a comparative effectiveness study. J Neurosurg. 2017;126(5):15231529.

37. Park SE, Kim JS, Park EK, et al. Direct versus indirect revascularization in the treatment of moyamoya disease. J Neurosurg. 2018;129(2):480-489.

\section{Disclosures}

Dr. Steinberg is a consultant for Qool Therapeutics, Peter Lazic US, NeuroSave, SanBio, and Zeiss. Dr. Heit is a consultant for Medtronic and MicroVention.

\section{Author Contributions}

Conception and design: Steinberg, Nielsen. Acquisition of data: Steinberg, Nielsen, Bell-Stephens, Heit. Analysis and interpretation of data: Nielsen, Abhinav, Sussman, Han, Weng. 
Drafting the article: Nielsen, Abhinav, Sussman, Weng. Critically revising the article: Steinberg, Nielsen, Abhinav, Sussman, Han, Weng, Heit. Reviewed submitted version of manuscript: all authors. Approved the final version of the manuscript on behalf of all authors: Steinberg. Statistical analysis: Nielsen, Han, Weng. Administrative/technical/material support: Steinberg. Study supervision: Steinberg, Nielsen.

\section{Supplemental Information}

\section{Online-Only Content}

Supplemental material is available with the online version of the article.

Supplementary Table 1. https://thejns.org/doi/suppl/10.3171/ 2020.3.JNS192847.

\section{Previous Presentations}

Part of this work comprised an oral presentation at the 67th Scandinavian Neurosurgical Congress and the 38th Herbert Olivecrona Symposium held in Stockholm, Sweden, on May 19-22, 2019.

\section{Correspondence}

Gary K. Steinberg: Stanford University School of Medicine, Stanford,CA. gsteinberg@stanford.edu. 